\title{
Plataforma colaborativa multimídia para apoio ao diagnóstico de lesões bucais em ambientes de teleodontologia
}

\author{
Maria Inês Meurer*, Aldo von Wangenheim**, Caroline Zimmermann***, Douglas Dyllon \\ Jeronimo de Macedo****, Andre Puel*****, Martin Prüsse*****, Tiago de Holanda Cunha \\ Nóbrega*****. \\ * Professora do Departamento de Patologia, Universidade Federal de Santa \\ Catarina (UFSC). \\ ** Professor do Departamento de Informática e Estatística, UFSC. \\ *** Mestranda do Programa de Pós-Graduação em Odontologia, UFSC. \\ **** Doutorando do Programa de Pós-Graduação em Engenharia e Gestão do \\ Conhecimento, UFSC. \\ ****** Pesquisador do Instituto Brasileiro para Convergência Digital - INCoD, UFSC.
}

\section{RESUMO}

Este artigo tem por objetivo apresentar uma solução tecnológica desenvolvida para permitir atividades colaborativas de diagnóstico de lesões bucais em ambientes de Teleodontologia. Trata-se de uma plataformatotalmente baseada na web e que permite o gerenciamento e compartilhamento de imagens clínicas, radiográficas e histopatológicas, dispensando a instalação de qualquer programa adicional no computador do usuário. Como suporte à colaboração através do navegador, as ações efetuadas por um usuário são replicadas e visíveis ao outro usuário conectado, sendo possível a discussão e troca de informações sobre o caso através de chat e/ou áudiovideoconferência.Ferramentas de Telessaúde podem oferecer diferentes oportunidades para assistência e educação em Odontologia. Ao permitir a visualização de diferentes aspectos da doença do paciente, a solução aqui apresentada tem potencial para viabilizar: (1) a troca de informações entre profissionais de saúde atuantes em distintos níveis de assistência e (2) a tutoria, à dis- tância, nos diferentes níveis de formação (graduação, pós-graduação e educação continuada).

Descritores: Teleodontologia; Telemedicina; Diagnóstico.

Projeto financiado pela Financiadora de Estudos e Projetos - FINEP.

\section{INTRODUÇÃO}

No Brasil, historicamente os serviços de atenção à saúde concentraram-se em torno de centros populacionais mais desenvolvidos socioeconomicamente; regiões fronteiriças, centros populacionais menores e áreas com menor índice de urbanização possuem acesso insuficiente aos serviços públicos de saúde ${ }^{11,16,18}$. Mesmo em regiões com boa distribuição populacional, bons indicadores sociais e/ou concentração demográfica relevante este fenômeno é ainda observável ${ }^{5,6,11,20}$.

Em decorrência dessa distribuição irregular, frequentemente os pacientes necessitam viajar longas distâncias para reali- 
zar determinados exames ou se submeter a atendimentos e tratamentos especializados. No Sistema Único de Saúde, o tratamento fora de domicílio é disponibilizado e pago pelo Estado ${ }^{2}$, não sendo apenas um fator de custo direto, mas também uma situação que pode acarretar o agravamento da condição de saúde do paciente. Além disso, a descontinuidade e fragmentação das informações do paciente entre as frentes assistenciais e ao longo do processo assistencial contribuem para a diminuição do potencial de resolutividade do sistema público de saúde como um todo ${ }^{8,10}$.

Tal cenário também se apresenta no diagnóstico e tratamento das doenças pertinentes ao complexo bucomaxilofacial, que podem apresentar desde um pequeno impacto local até um grande impacto na integralidade da saúde do paciente. Há, por exemplo, tumores benignos que apresentam comportamento agressivo, resultando em tratamentos cirúrgicos efetuados em ambiente hospitalar e que podem resultar em consequências funcionais e estéticas graves. Algumas lesões bucais são manifestações de doenças sistêmicas, permitindo o diagnóstico destas últimas-como é o caso das manifestações bucais da Síndrome da Imunodeficiência Adquirida e outras doenças sexualmente transmissíveis, do diabetes, de algumas doenças hematológicas e dermatológicas, entre outras. O câncer de boca destaca-se pelo seu impacto tanto na vida do paciente quanto nos custos de tratamento para o sistema de saúde: além da alta mortalidade de indivíduos na faixa etária economicamente ativa - a sobrevida em 5 anos gira em torno de $50 \%{ }^{3}$-,deve-se considerar que o seu tratamento geralmente implica em mutilações que, muitas vezes, inabilitam o paciente para a reintegração familiar, social e profissional, temporária ou definitivamente $^{12,13}$. A maioria dos casos de câncer de boca é diagnosticada tardiamente ${ }^{17}$, embora seja conhecido o perfil dos pacientes de risco (tabagistas crônicos, principalmente) e existam alterações que tendem a preceder as lesões de câncer (na cavidade bucal destacam-se as leucoplasias, eritroplasias e o líquen plano; nos lábios, a queilite actínica) ${ }^{1}$. Neste sentido, o cirurgião-dentista é um profissional chave no diagnóstico e tratamento das lesões bucais.

A Odontologia brasileira é reconhecida mundialmente pela excelência técnica em devolver aos pacientes - com estética e função - os elementos dentais perdidos,e os cirurgiões-dentistas brasileiros, de forma geral, estão mais centrados no diagnóstico das doenças que acometem dentes e periodonto. Pacientes portadores de doenças não relacionadas à cárie e/ou doença periodontal são frequentemente referenciados ao nível secundário de atenção, sendo o processo de diagnóstico e tratamento da doença conduzido por diferentes profissionais da Odontologia, como estomatologistas, radiologistas, patologistas bucais e cirurgiões bucomaxilofaciais. Na maioria dos estados brasileiros são poucos os profissionais com formação especialista a quem pacientes com essas lesões podem ser referenciados (Tabela 1).

Tabela 1 - Número de profissionais registrados no Conselho Federal de Odontologia, por especialidade, ressaltando-se a quantidade relativamente pequena de profissionais com formação específica nas áreas do diagnóstico e tratamento de lesões bucais (excetuando-se cárie e doença periodontal) 


\begin{tabular}{lc}
\hline Especialidade registrada no CFO & Números de especialistas \\
\hline Endodontia & 13.128 \\
Prótese & 10.030 \\
Periodontia & 9.175 \\
Implantodontia & 8.910 \\
Dentística & 5.893 \\
Radiologia & 4.986 \\
Cirurgia Bucomaxilofacial & 4.714 \\
Estomatologia & 827 \\
Patologia Bucal & 408 \\
\hline
\end{tabular}

Fonte: Conselho Federal de Odontologia, 2013..Disponível em http:/cfo.org.br/servicos-econsultas/Dados-estatisticos, acesso em 08 janeiro de 2013.

Quando o paciente com lesão bucal é referenciado, os profissionais envolvidos noseu atendimento precisam ter acesso a informações sobre os aspectos clínicos, radiológicos e histopatológicos das lesões, dados estes nem sempre disponíveis. Há casos, ainda, em que a troca de informações e opiniões pode ser desejável, como nas doenças raras ou nos casos de maior complexidade diagnóstica.Para Nobre et al $(2007)^{14}$, “o intercâmbio eficiente de informação entre profissionais de saúde pode economizar tempo e dinheiro, proporcionar maior efetividade clínica, melhorar a continuidade e a qualidade da assistência, assim como facilitar as atividades de gestão em sistemas de saúde públicos e privados".

A Telemedicina tem entre seus principais objetivos tornar os serviços de saúde acessíveis para populações que vivem fora dos grandes centros urbanos, agilizando o processo de diagnóstico. Assim, é desejável que ferramentas de telemedicina permitam o acesso à história da doença do paciente, a visualização e interação com exames que envolvam imagens e, ainda, a comunicação entre os usuários, permitindo a colaboração entre os mesmos - tudo isto de forma facilmente acessível em qualquer lugar.Em atenção a este cenário, desenvol- veu-se uma solução tecnológica integrada em uma única plataforma e destinada ao gerenciamento de imagens clínicas, radiológicas e histopatológicas,e que permitea discussão colaborativa entre profissionais de saúde atuantes nas distintas fases da assistência.A plataforma também tem potencial para uso em ambientes educacionais à distância.

Os resultados aqui relatados são iniciais. Os autores optaram por apresentá-los considerando o ainda reduzido número de trabalhos tecnológicos em Teleodontologia no Brasil, a potencial contribuição desta solução em ambientes de Telessaúde e seu eventual impacto sobre a cadeia assistencial do Sistema Único de Saúde.

\section{CARACTERÍSTICAS DA SOLUÇÃO TECNOLÓGICA PROPOSTA}

A plataforma é totalmente baseada na web, podendo ser utilizados os navegadores Firefox e Chrome para sua execução. O desenvolvimento foi baseado em padrões e tecnologias abertas, não havendo a necessidade de instalação de softwares específicos- sejam eles livres ou comerciais - o que viabiliza a utilização a partir de qualquer computador conectado à Internet. 
O acesso às imagens é efetuado através de uma "plataforma-mãe" que apresenta as funcionalidades de um PACS (Picture Archiving and Communication System) - um conjunto de tecnologias usado particularmente no âmbito da Radiologia, e que permite o arquivamento seguro e ordenado de imagens obtidas por diferentes equipamentos,podendo ser acessadas através de redes de computadores ${ }^{7}$.

$\mathrm{O}$ acesso à plataforma é restrito (usuário e senha), e segue os mesmos moldes da Rede Catarinense de Telemedicina e Telessaúde no que se refere aos aspectos de segurança e confiabilidade no trânsito de documentos clínicos eletrônicos baseados na internet ${ }^{9,14,19}$. Seu funcionamento está baseado no envio das imagens clínicas, radiológicas e histopatológicas para um servidor, permitindo posteriormente o acesso remoto pelos profissionais envolvidos no caso, sendo possível a visualização e processamento das imagens, bem como a discussão (colaboração) de forma síncrona.

O módulo de visualização é denominado DIMP (Digital Imaging Manipulation Program). Ao se carregar as imagens no DIMP a partir do servidor, o sistema verifica se já existe algum usuário examinando o mesmo conjunto de imagens e, em caso positivo, anuncia a entrada do novo usuário, estando disponíveis as ferramentas de chat e áudio-videoconferência para a colaboração remota. A partir do estabelecimento da conexão e abertura das imagens no DIMP, todas as ações efetuadas por um usuário (utilizando mouse e teclado) são imediatamente replicadas e visíveis aooutro usuário conectado.

A seguir serão descritas as principais funcionalidades da plataforma.

\subsection{Visualização e processamento de imagens radiológicas}

Esta funcionalidade já está estabelecida em ambiente real, sendo usada como rotina na visualização de exames radiológicos na Rede Catarinense de Telemedicina e Telessaúde. Imagens obtidas digitalmente como é o caso dos equipamentos radiológicos digitais, de tomografia computadorizada (TC) e ressonância magnética (RM) - são armazenadas no formato DICOM (formato de imagem próprio de exames radiológi$\cos )$.

Quando o profissional acessa a plataforma, é apresentada a listagem dos pacientes (Figura 1)aos quais ele pode ter acesso. Após identificar as imagens a serem acessadas, o profissional solicita que sejam abertas no visualizador DIMP (Figura 2). Navegadores de internet não são preparados para visualização de imagens DICOM, de forma que foi necessário o desenvolvimento de um procedimento especial para permitir seu carregamento.

Figura 1 - Interface de usuário, apresentando a listagem de pacientes aos quais o profissional pode ter acesso 


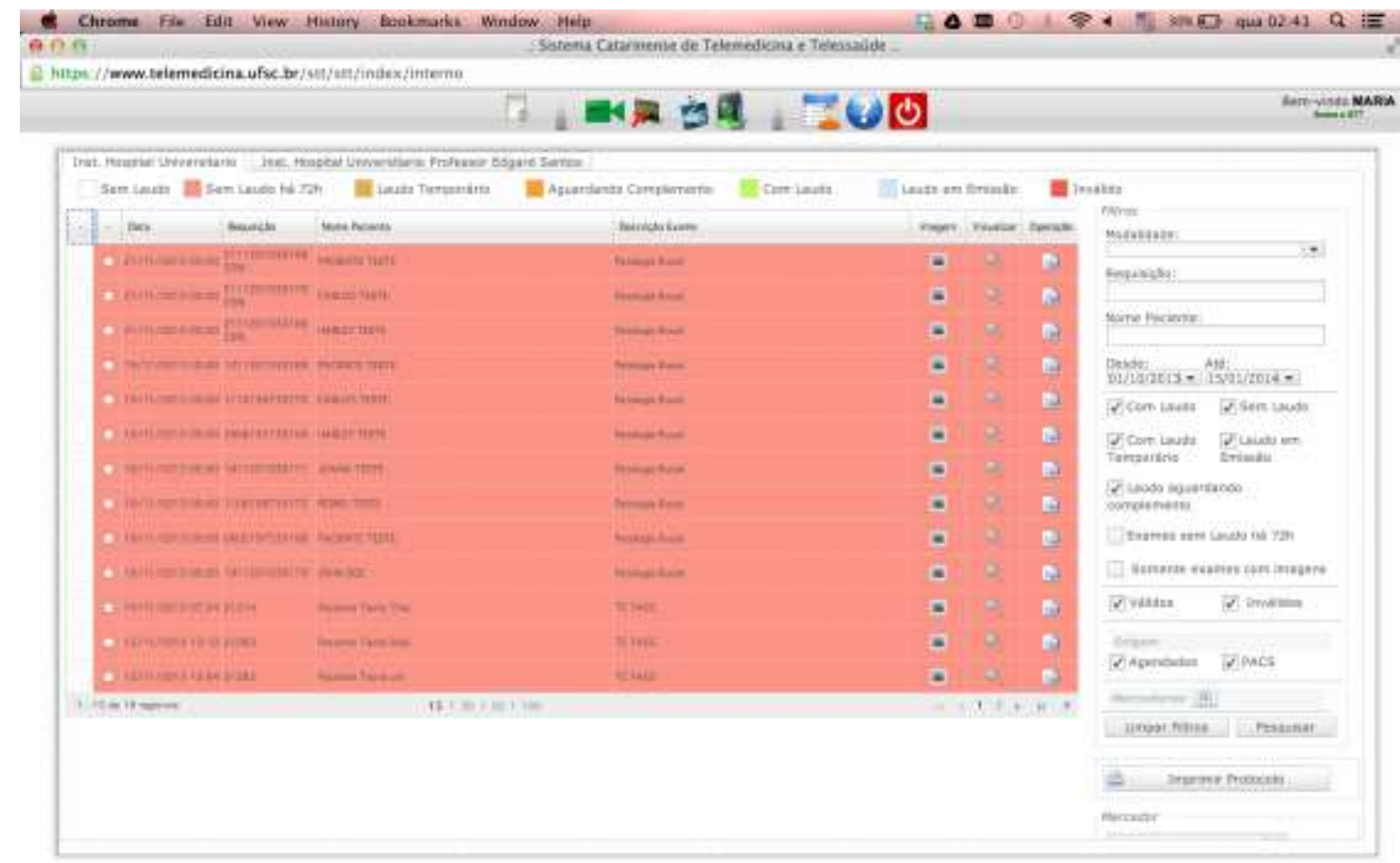

Figura 2 - Interface de usuário do DIMP, apresentando uma imagem de tomografia computadorizada de face. As funcionalidades básicas de programas para visualização e processamento dessas imagens estão disponíveis (sequência de botões acima e à direita). Podese ainda observar a janela para chat no canto superior esquerdo.

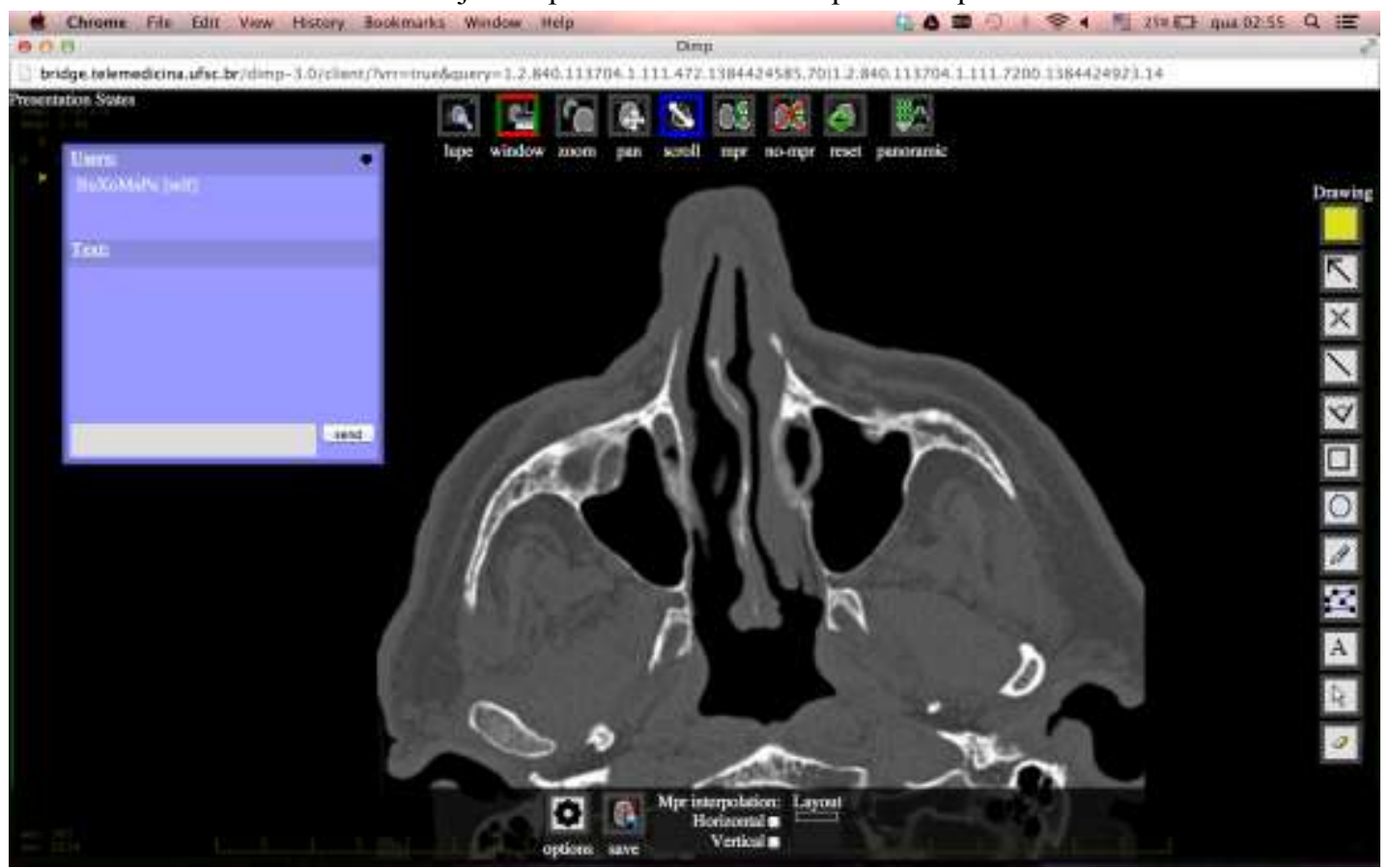

O DIMP oferece as funcionalidades fundamentais de uma workstation radiológica para visualização e análise desse tipo de imagem ${ }^{15}$, como zoom, janelamento (variação nos tons de cinza da imagem), men- surações lineares e de densidade tomográfica, entre outros. Além disso, o DIMP permite a execução de reconstruções multiplanares (axiais, coronais e sagitais) (Figura 3). Está em fase avançada de desenvolvi- 
mento a ferramenta para processamento tridimensional (3D).

$\mathrm{Na}$ Odontologia, a análise de séries de imagens de tomografia computadorizada requer muitas vezes reconstruções diferenciadas, denominadas panorâmicas e oblíquas (ou paraxiais). A reconstrução panorâmica objetiva a visualização de todo o arco dental, gerada como uma única imagem plana de uma seção com forma elíptica. No DIMP, o plano elíptico de reconstrução é gerado pelo usuário, através do posicionamento de pontos de controle; o software então interpola uma curva que passa pelos pontos de controle, e gera a reconstrução. As reconstruções oblíquas envolvem a geração de secções transversais ao longo da curva definida pelo usuário, criando um conjunto de sub-imagens que permite a visualização vestíbulolingual/palatal dos processos alveolares de maxila e mandíbula. Na Figura 3, podem ser observadas reconstruções panorâmica e oblíquas, sendo possível a navegação pelo conjunto de reconstruções. Também está implementada a ferramenta de mensuração sobre estas reconstruções (que ainda precisa ser validada quanto à sua precisão).

Imagens obtidas por tomógrafos computadorizados de feixe cônico (TCFC) também podem ser também visualizadas no DIMP. Infelizmente, vários equipamentos de TCFC ainda produzem imagens em DICOM fora do padrão, sendo necessárias adequações no sistema para que tais imagens possam ser visualizadas e processadas no DIMP.

Figura 3 - Reconstruções multiplanares (à esquerda) e reconstruções panorâmica e oblíquas (à direita) a partir de imagens de tomografia computadorizada

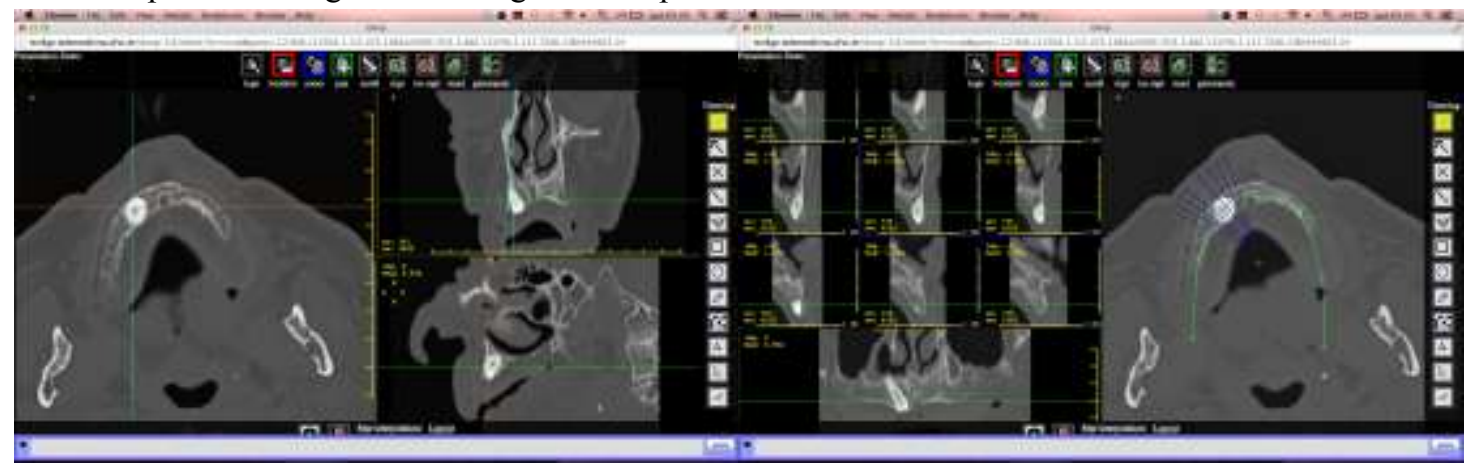

\subsection{Visualização de imagens clínicas}

Imagens clínicas também podem ser visualizadas no DIMP. O formato .JPG é o mais comumente utilizado nas imagens obtidas por equipamentos fotográficos digitais, mas o sistema suporta outros formatos. Imagens radiográficas que tenham sido digitalizadas por equipamentos não dedicados, como escâneres, máquinasfotográficas e smartphones, também podem ser visualizadas (Figura 4).

Figura 4 - Fotografia intrabucal e radiografia panorâmica de um caso de lesão cística em maxila, visualizadas no DIMP. A radiografia foi digitalizada com máquina fotográfica semiprofissional. Algumas ferra- 
mentas disponíveis para as imagens radiológicas também podem ser utilizadas, permitindo, por exemplo, o ajuste de brilho e contrasteou do zoom da imagem

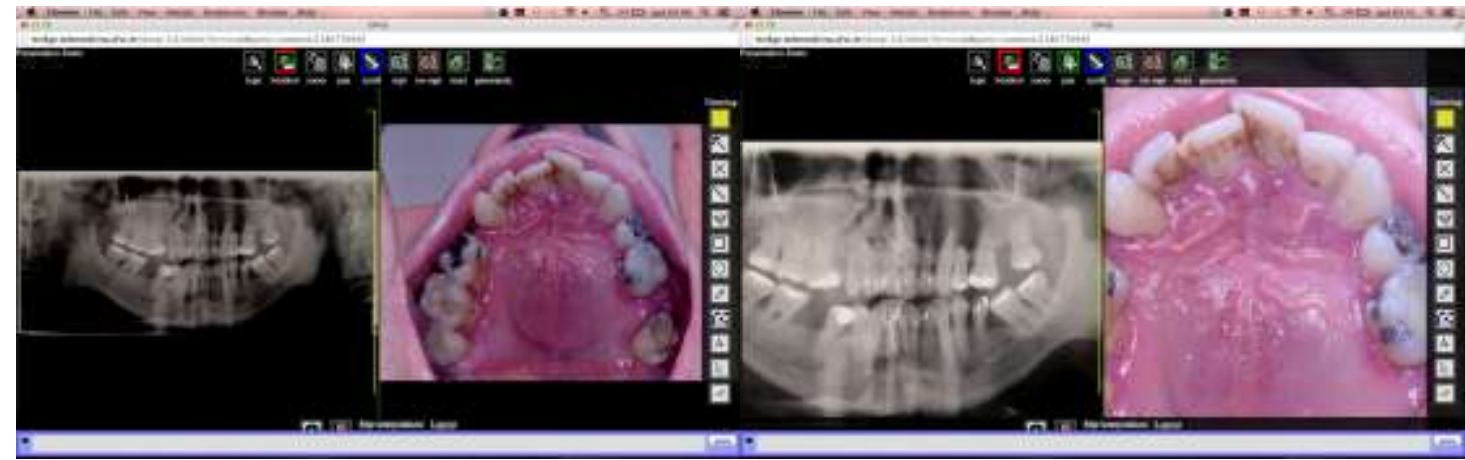

\subsection{Imagens histopatológicas}

Para o compartilhamento de imagens histopatológicas, a solução mais lógica seria a simples digitalização utilizando câmera fotográfica digital acoplada ao microscópio. No entanto, a amostra tecidual é geralmente muito maior que o campo de visão que o microscópio oferece sob amplificação, e a digitalização de subáreas individuais não é tida pelos patologistas como suficiente para análise à distância, sendo essencial a visualização da lâmina como um todo, de forma similar ao que ocorre com a amostra física.

Há escâneres dedicados à digitalização de lâminas, como os equipamentos comercializados por empresas como Aperio (www.aperio.com), Nikon (http://www.nikoninstruments.com/pr_BR/ Products/Digital-Pathology), Philips (http://www.research.philips.com/initiative s/digitalpathology/) e PathXL (http://www.pathxl.com/about-pathxl). No entanto, são equipamentos de alto custo, tendo sido inviável a sua aquisição com os recursos disponíveis para a execução do projeto. Assim, optou-se por um sistema automatizado, composto por microscópio óptico convencional trinocular acoplado a motor de passo (platina motorizada XYZ) e câmera digital.
Embora possa parecer uma solução de simples efetivação, uma análise mais apurada revelou haver incompatibilidade entre os diferentes componentes do sistema, oferecidos por diferentes fornecedores $^{\mathrm{i}}$. Evidenciou-se que alguns equipamentos não apresentavam interoperabilidade; e noutras configurações cogitadas, o microscópio ficaria dedicado a esta única função. Considerando os objetivos do projeto, a interoperabilidade, a disponibilidade de recursos e a decisão de não tornar o microscópio dedicado a este único fim, optou-se pelo seguinte conjunto (Figura 5): microscópio trinocular Primo Star (Zeiss) + Platina Motorizada XYZ modelo OptiScan II ES103Z (Prior Scientific) + Adaptador C-mount + Câmera digital infinity 11 C (Qimaging/Lumenera) + Software Image-Pro Plus Plus v7.0 (Media Cybernetics). Acrescentou-se ao conjunto uma mesa antivibratória (Kinetic Systems) para reduzir o efeito de vibrações mecânicas ambientais que interferem no funcionamento do sistema motorizado.A visualização das imagens foi integrada ao DIMP (Figuras 6 e 7). Também foi desenvolvido

\footnotetext{
'Foram avaliados microscópios das fabricantes Leica, Olympus, Nikon e Zeiss. Para automação da aquisição, foram avaliadas as opções dos fabricantes Prior, Marzhauser, Zeiss, Objective Imaging e Trofa, bem como sistemas de câmeras e softwares (Dyno Eye, EM 200-F, Cell Profiler, ImageJ, Fiji e $\mu$ Manager).
}

Revista da ABENO • 13(2): 13-26, 2013 
o suporte web necessário à visualização das imagens, sendo equivalente aos programas para exploração de mapas (como o Google Maps), permitindo aproxima- ção/distanciamento e navegação, além de ajustes de brilho e contraste da imagem, se necessário.

Figura 5 - Sistema de aquisição de imagens histopatológicas acoplado ao computador.

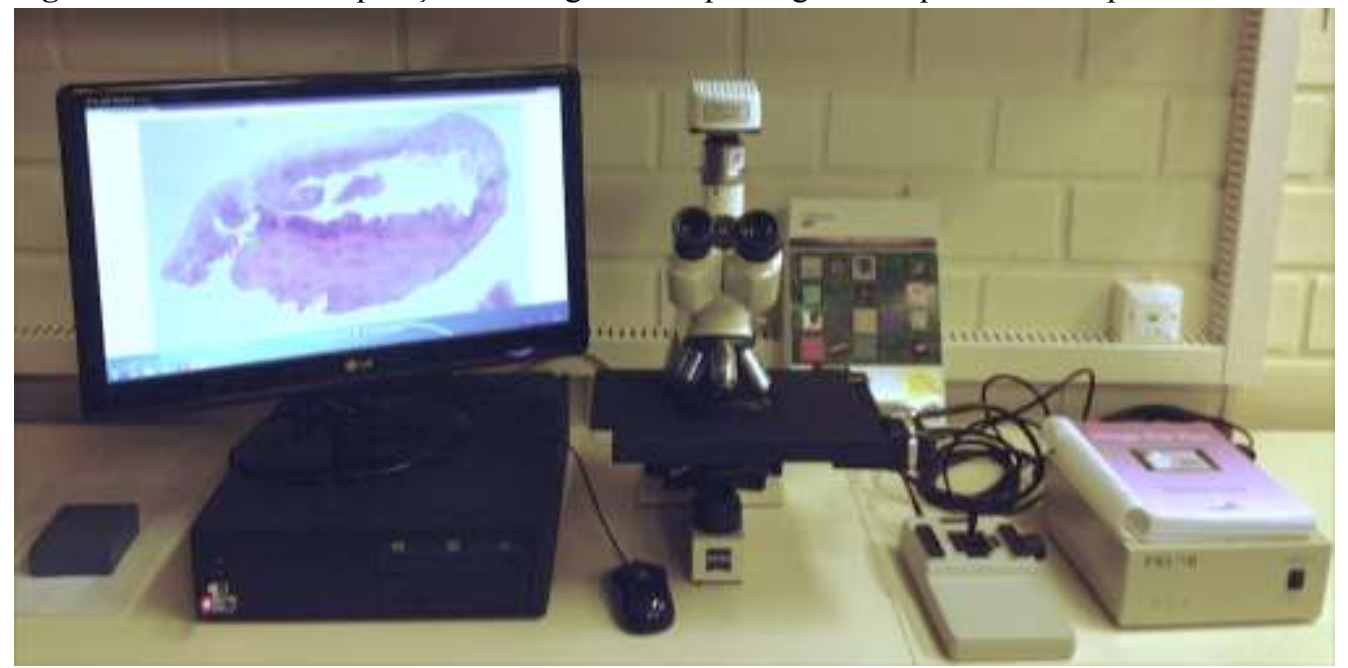

Figura 6 - Imagem histopatológica visualizada no DIMP utilizando o navegador Chrome

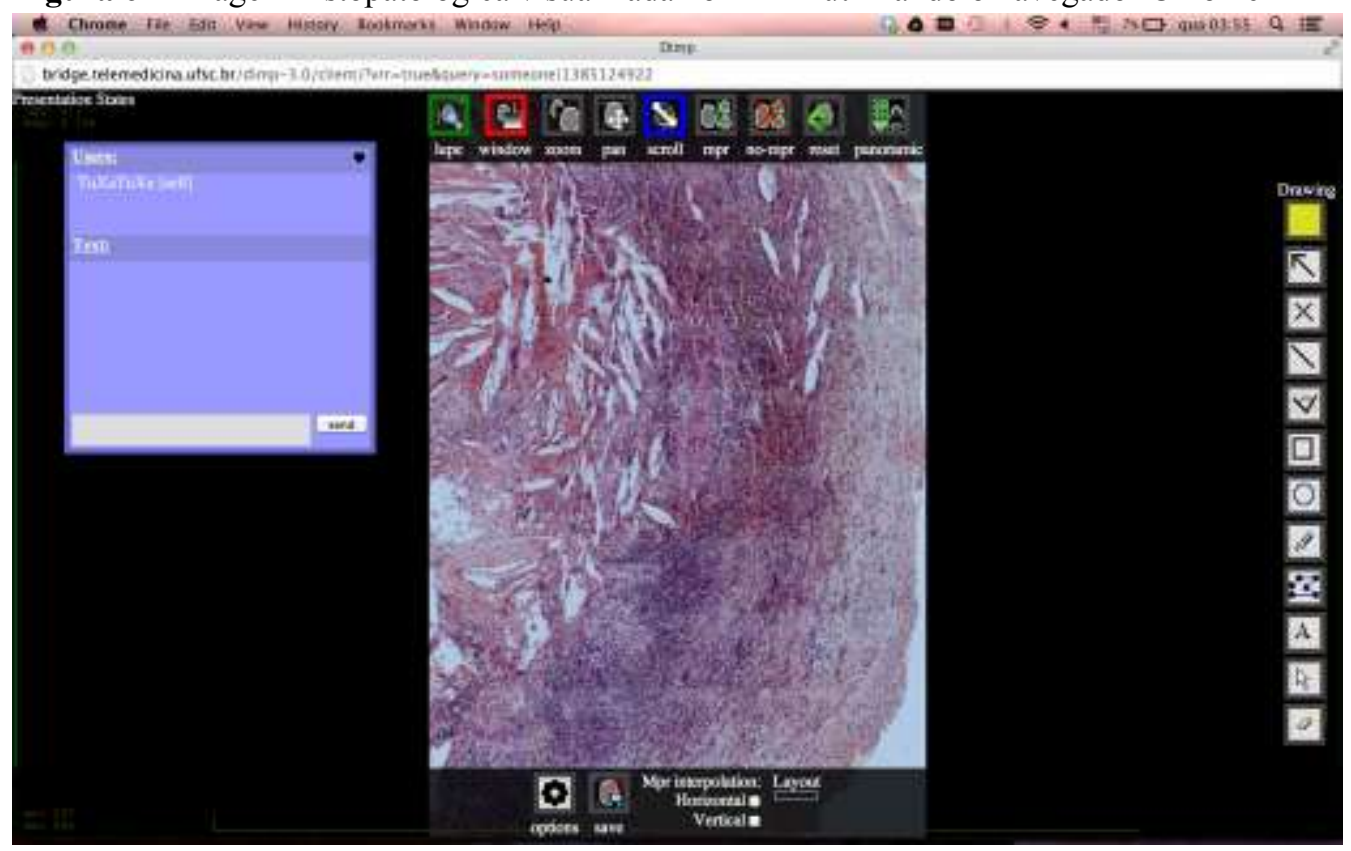

Figura 7 - Mesmo caso da figura 6, demonstrando-se aqui a utilização da ferramenta de zoom para ampliação, bem como outras que podem ser utilizadas durante a discussão para indicação de áreas de interesse (neste caso, círculo e seta amarelos) 


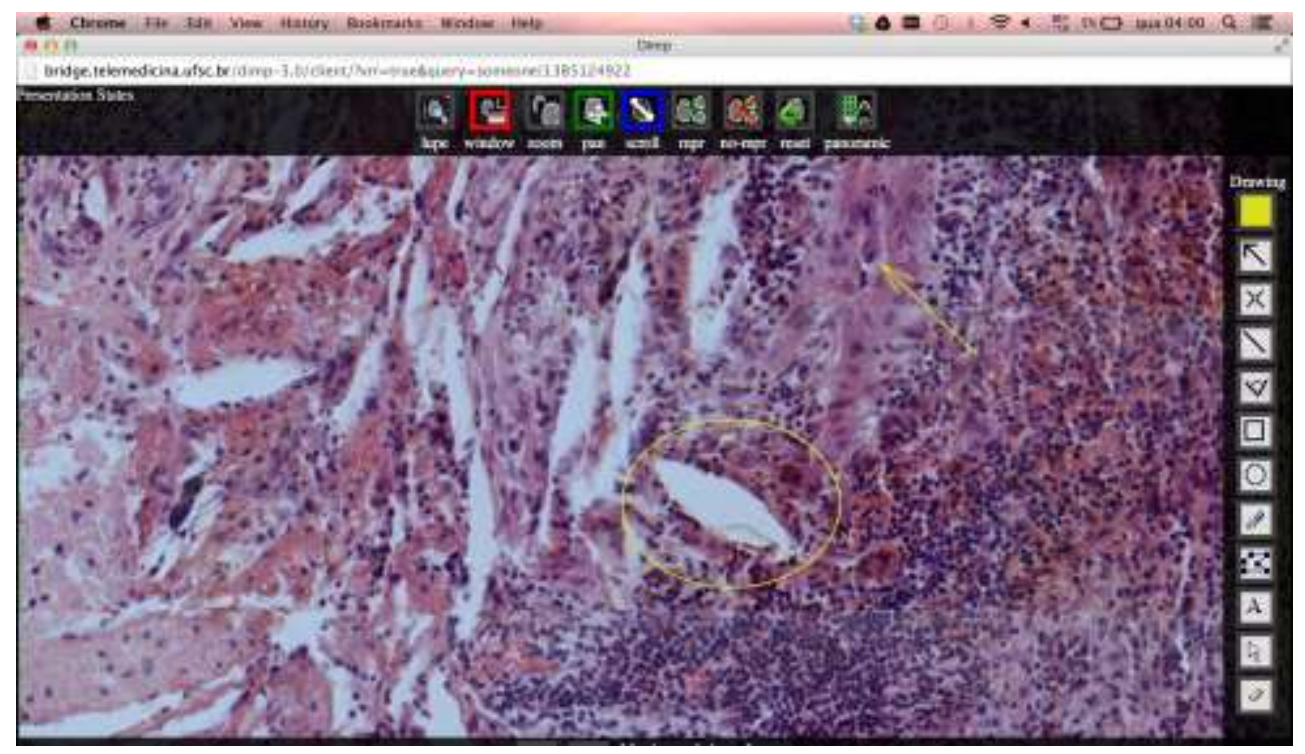

\subsection{Suporte à colaboração}

Por suporte à colaboração entendese a disponibilidade de ferramentas que permitam a um usuário do sistema compartilhar por chat e/ou voz/vídeo as informações do caso e suas impressões sobre o diagnóstico, bem como efetuar determinadas ações em tela que possam ser visualizadas por outro usuário localizado remotamente.

Da mesma forma que as demais ferramentas desenvolvidas, este processo colaborativo está integrado à plataforma. Foram implementadas duas formas de comunicação: áudio/videoconferência e chat. No caso da videoconferência (Figura 8), quando o usuário entra na plataforma ele tem a opção de iniciar uma sessão de videoconferência com outro usuário conectado. Após estabelecida a conexão, ambos os usuários escolhem o mesmo exame para compartilhar, mantendo-se ativa a áudio/videoconferência em curso. A segunda forma de comunicação é por chat. Neste caso, assim que um usuário solicita a abertura de um exame no visualizador DIMP, o sistema verifica se já existe um outro usuário examinando o mesmo conjunto de imagens e, em caso positivo, anuncia a sua entrada através de uma janela de chat (Figuras 2 e 6$)$.

Independente da forma de comunicação utilizada (chat ou áudio/videoconferência), a partir do momento que ambos os usuários estão conectados e visualizando um mesmo exame, as ações efetuadas por um são imediatamente replicadas e visíveis ao outro. Estas duas capacidades (troca de informações por chat ou áudio-videoconferência + compartilhamento de ações efetuadas por mouse e teclado) permitem efetivamente a discussão dos casos utilizando o conjunto de dados disponível (fotos clínicas, imagens radiológicas e/ou imagens histopatológicas).

Figura 8 - Interface do usuário na janela principal da plataforma, onde pode ser observada janela de videoconferência (canto inferior direito). 


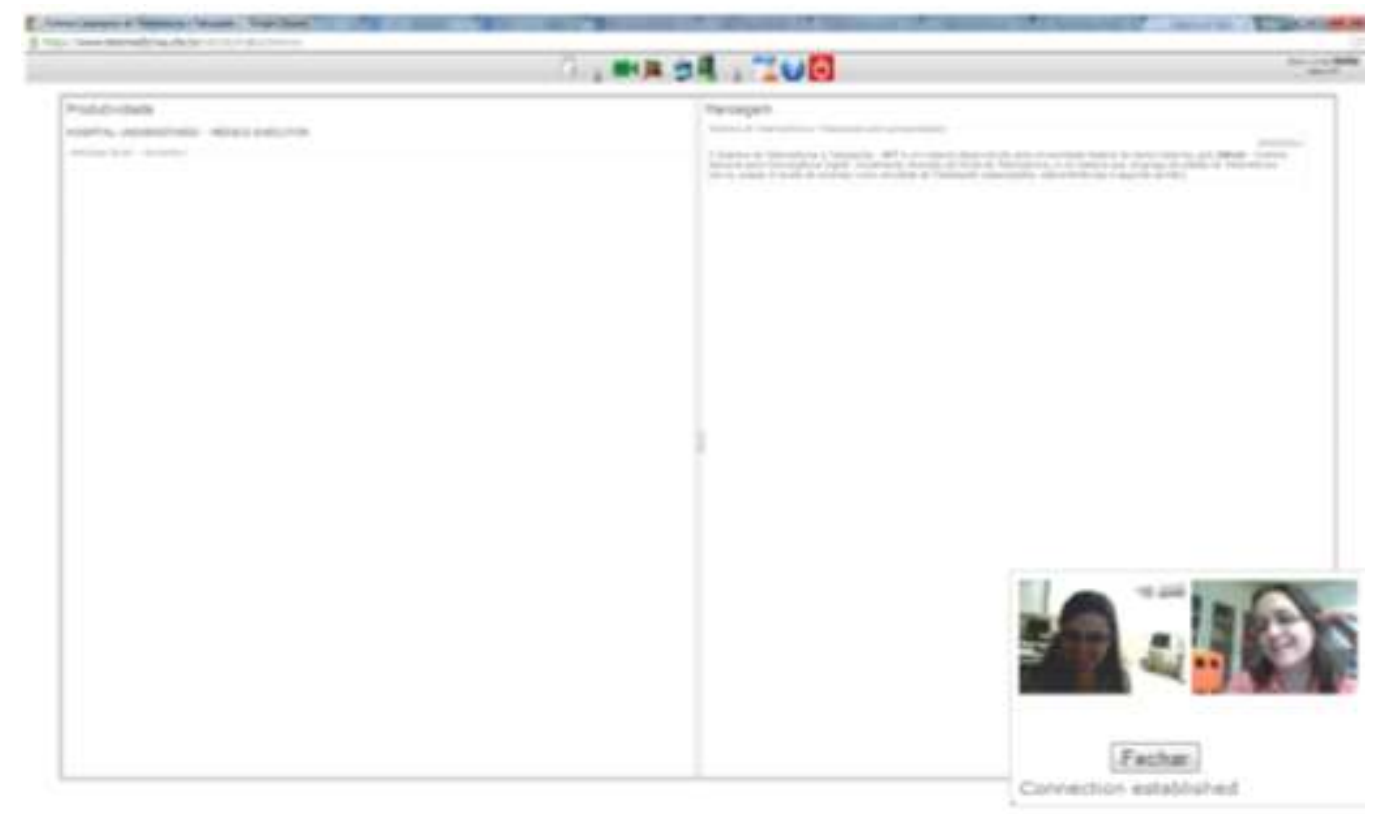

\subsection{Sistema de acervo digital}

No decorrer do desenvolvimento, verificou-se a necessidade de dispor de um sistema de acervo para gerenciamento e arquivamento das imagens, também de acesso restrito. No acervo são inseridas as imagens e dados correlatos. Há campos para registro de data da imagem, história clínica e tipo de exame. Podem ser incluídos diversos formatos de imagem, individualmente ou de forma seriada (como é o caso das imagens de exames de $\mathrm{TC}$ ou $\mathrm{RM}$ ). Adicionalmente, foram inseridos campos para registro de hipóteses de diagnóstico, diagnóstico clínico (presuntivo) e histopatológico - nos dois últimos com consulta direta ao CID-10. Está em fase de implementação a ferramenta de busca para fins de relatórios (tipos de lesões, incidência, perfis de ocorrência de determinada doença em determinada população, etc.). Também estão em discussão os métodos e processos para anonimização de dados e/ou imagens, visando a sua utilização nos processos colaborativos.

\section{TESTES DE VALIDAÇÃO}

Participaram dos testes de validação profissionais das áreas de Estomatologia, Radiologia Odontológica, Patologia Bucal e Cirurgia Bucomaxilofacial. Estão sendo iniciados alguns testes junto a profissionais vinculados a Unidades Básicas de Saúde e que tenham alguma experiência no uso de ferramentas web no âmbito do Programa Telessaúde Brasil.

A metodologia de validação escolhida para avaliar o impacto destas ferramentas sobre a prática dos profissionais foi baseada na experiência de usuário. A lógica por detrás dessa decisão é a de que, se a ferramenta proposta é um auxiliar na execução do trabalho deste profissional, mas existem outras formas mais tradicionais de realizá-lo é, no fim de contas, a experiência do usuário que decidirá se ele a utilizará no futuro - ou seja, se ele considerou fácil, agradável, produtivo e frutífero utilizar a ferramenta proposta.

Foi utilizado um instrumento tradicional e bem estabelecido na literatura e na comunidade de Usabilidade em Computação para avaliação da experiência do usuário: a Escala de Usabilidade de Sistemas (SUS -System Usability Scale) ${ }^{4}$. Este ins- 
trumento utiliza um formulário a ser preenchido pelo usuário, que busca coletar suas impressões após o uso do software, resultando em um escore final.Os escores numé- ricos foram "traduzidos" em adjetivos que expressam a usabilidade do software ${ }^{1}$, conforme a Tabela 2.

Tabela 2 - Conversão dos valores da escala SUS em adjetivos que expressam subjetivamente a usabilidade de um sistema

\begin{tabular}{ccc}
\hline Escore & Desvio padrão & Adjetivação \\
\hline 90,9 & 13,4 & "o melhor imaginável" \\
85,5 & 10,4 & "excelente" \\
71,4 & 11,6 & "bom" \\
50,9 & 13,8 & "ok" \\
35,7 & 12,6 & "ruim" \\
20,3 & 11,3 & "muito ruim" \\
12,5 & 13,1 & "inconcebível" \\
\hline
\end{tabular}

Fonte: Bangor, Kortum e Miller, 2008.

Os testes de validação efetuados até o momento contaram com a participação de onze profissionais que atuam nas especialidades odontológicas envolvidas no diagnós- tico e tratamento de lesões bucais, conforme Tabela 3. A média obtida $(88,2)$ indica que o sistema foi tido como excelente pelos usuários durante os testes de validação.

Tabela 3 - Valores da escala SUS (System Usability Scale) para avaliação do sistema, a partir das respostas de profissionais das especialidades envolvidas no diagnóstico e tratamento de lesões bucais.

\begin{tabular}{lcccccccccccc}
\hline Usuário & $\mathbf{0 1}$ & $\mathbf{0 2}$ & $\mathbf{0 3}$ & $\mathbf{0 4}$ & $\mathbf{0 5}$ & $\mathbf{0 6}$ & $\mathbf{0 7}$ & $\mathbf{0 8}$ & $\mathbf{0 9}$ & $\mathbf{1 0}$ & $\mathbf{1 1}$ & Média \\
\hline Especialidade(s)* & $\mathrm{E}$ & $\mathrm{E}$ & $\mathrm{E}$ & $\mathrm{P}$ & $\mathrm{P}$ & $\mathrm{R}$ & $\mathrm{P}$ & $\mathrm{E}$ & $\mathrm{E}$ & $\mathrm{E}$ & $\mathrm{E}$ & \\
& $\mathrm{R}$ & $\mathrm{P}$ & $\mathrm{P}$ & & & $\mathrm{C}$ & & $\mathrm{R}$ & $\mathrm{R}$ & & & \\
& 85 & 95 & 100 & 100 & 87,5 & 82,5 & 82,5 & 80 & 85 & 95 & 77,5 & $\mathbf{8 8 , 2}$ \\
\hline
\end{tabular}

Especialidades: (E) Estomatologia; (R) Radiologia; (P) Patologia Oral; (C) Cirurgia Bucomaxilofacial.

* Alguns profissionais são especialistas em mais de uma área.

\section{CONSIDERAÇÕES FINAIS E PERS- PECTIVAS FUTURAS}

Os resultados dos testes de usabilidade iniciais indicam que a plataforma baseada na web se adapta às necessidades dos potenciais usuários. Já estão sendo analisa das as possibilidades de interação da plataforma com ambientes virtuais de aprendizagem como o Moodle.

Embora a plataforma possa ser acessada de qualquer computador, a interface baseada em gestos do mouse impede seu uso em smartphones e tablets. Está em avaliação o desenvolvimento de uma versão 
adaptada a este perfil de dispositivo móvel, estando prevista a repetição comparativa da avaliação de usabilidade aqui apresentada.

A equipe de coordenação e desenvolvimento acredita que uma solução tecnológica que permita a organização e visualização de diferentes aspectos da doença do paciente, bem como a discussão desses da-

\section{REFERÊNCIAS}

1. Bangor A, Kortum PT, Miller JT. An empirical evaluation of the System Usability Scale.Int $J$ Human-Comput Interact. 2008;24(6):574-94.

2. Brasil. Ministério da Saúde. Portaria $/ S A S / N^{o}$ 055, 1999. Disponível em:http://dtr2001.saude.gov.br/sas/PO RTARIAS/Port99/PT-055.html.

3. Brasil. Ministério da Saúde. Secretaria de Assistência à Saúde. Instituto Nacional de Câncer - INCA. Falando Sobre Câncer da Boca. Rio de Janeiro: INCA; 2002.52 p. Disponível em http://bvsms.saude.gov.br/bvs/publicac oes/falando_sobre_cancer_boca.pdf.

4. Brooke J. SUS: a "quick and dirty" usability scale. In: Jordan PW, Thomas B, Weerdmeester BA, McClelland AL, editors. Usability Evaluation in Industry. London: Taylor and Francis;1996. P. 189-94.

5. Conselho Federal De Medicina. Demografia Médica no Brasil. Volume 1: Dados gerais e descrições de desigualdades. São Paulo: Conselho Regional de Medicina do Estado de São Paulo / Conselho Federal de Medicina; 2011.118p. Disponível em: dos de forma organizada e colaborativa, potencialmente viabilizará: 1) a troca de informações entre profissionais de saúde atuantes em distintos níveis de assistência (suporte assistencial) e 2) a tutoria, à distância, nos diferentes níveis de formação (graduação, pós-graduação e educação continuada).

http://www.cremesp.org.br/pdfs/demog rafia_2_dezembro.pdf.

6. Conselho Federal De Medicina. Demografia Médica no Brasil. Volume 2: Cenários e indicadores de distribuição. Coordenação: Mário Scheffer. São Paulo: Conselho Regional de Medicina do Estado de São Paulo / Conselho Federal de Medicina; 2013. 256p. Disponível em: http://www.cremesp.org.br/pdfs/Demo grafiaMedicaBrasilVol2.pdf.

7. De Backer AI, Mortelé KJ, De Keulenaer BL. Picture archiving and communication system - Part one: Filmless radiology and distance radiology. JBRBTR. 2004 Sep-Oct;87(5):234-41.

8. Dias MP, Giovanella L. Prontuário eletrônico - uma estratégia de coordenação entre a atenção primária e secundária à saúde no município de Belo Horizonte. RECIIS R Eletrde Com Inf Inov Saúde. 2013Ago;7(2)(Supp.). Disponível

em: http://www.reciis.icict.fiocruz.br/index. $\mathrm{php} /$ reciis/article/view/763/1638.

9. Giuliano IC, Barcellos Junior CL, von Wangenheim A, Coutinho MS. Issuing electrocardiographic reports remotely: experience of the telemedicine network of Santa Catarina. Arq Bras Cardiol. 2012 Nov;99(5):1023-30. Epub 2012 Oct.Disponível em: 
http://www.scielo.br/pdf/abc/v99n5/en _aop09012.pdf

10. Hartz ZMA, Contandriopoulos AP. Integralidade da atenção e integração de serviços de saúde: desafios para avaliar a implantação de um "sistema sem muros". Cad Saúde Pública [periódico na Internet]. 2004; 20( Suppl 2):S331S336. Disponível em: http://www.scielo.br/pdf/csp/v20s2/26. pdf

11. Instituto Brasileiro De Geografia E Estatística - IBGE. Estatísticas da Saúde. Assistência Médico-Sanitária 2009. Rio de Janeiro: IBGE, 2010. Disponível

em

http://www.ibge.gov.br/home/estatistic

a/populacao/condicaodevida/ams/2009/ ams2009.pdf

12. Irineu RA, Caprara A. Histórias de pacientes com câncer de boca e práticas de fumo em áreas rurais do nordeste brasileiro. Esc Anna Nery. 2008 Jul/Set;12(3):500-507. Disponível em $\mathrm{http}: / /$ revistaenfermagem.eean.edu.br/d etalhe_artigo.asp?id=349.

13. Mcintyre P. Don'tlook away: when the cure is disfiguring and disabling the care must be supportive and sustaining. CancerWorld.2007 Sept/Oct;20:58-62. Disponível em http://www.cancerworld.org/pdf/5460 patientvoice.pdf.

14. Nobre LF, Wangenheim, AV, Maia RS, Ferreira L, Marchiori E. Certificação digital de exames em telerradiologia: um alerta necessário. Radiol Bras. 2007 Dec;40(6): 415-421. Disponível em

http://www.rb.org.br/audiencia_pdf.asp

http://cetes.medicina.ufmg.br/revista/in dex.php/rlat/article/viewFile/51/168 ?aid2 $=1219 \&$ nomeArquivo $=\mathrm{v} 40 \mathrm{n} 6 \mathrm{a} 11$. pdf.

15. Nóbrega THC, Prüsse M, Meurer MI, Wangenheim, AV. Ferramenta web colaborativa para visualização e interação com imagens médicas. In: XIII Congresso Brasileiro de Informática em Saúde: Anais do XIII Congresso Brasileiro de Informática em Saúde; 2012 Nov 19-23; Curitiba, Brasil. São Paulo: Sociedade Brasileira de Informática em Saúde; 2012. p. 1-5. Disponível em http://www.sbis.org.br/cbis2012/arquiv os/273.pdf.

16. Rodrigues Filho J. A distribuição dos recursos de saúde no Brasil: a administração da desigualdade. Rev Adm Empres.1987 Set; 27(3). Disponível em http://www.scielo.br/pdf/rae/v27n3/v27 n3a07.pdf.

17. Santos LCO, Batista OM, Cangussu MCT. Characterization of oral cancer diagnostic delay in the state of Alagoas.Braz $\mathrm{J}$ Otorhinolaryngol. 2010 Jul/Ago;76(4): 416-22. Disponível em http://www.scielo.br/pdf/bjorl/v76n4/e n_v76n4a02.pdf.

18. Scatena JHG, Tanaka OY. Distribuição dos estabelecimentos de saúde no Brasil: para qual modelo caminhamos? RAP Rev Admin Pública. 1998 Jul/Ago;32(4): 7-25, 1998. Disponível em:

http://bibliotecadigital.fgv.br/ojs/index. php/rap/article/viewFile/7777/6377

19. Wangenheim AV, Barcellos Jr CL, Wagner HM, Gomes CC. Ways to implement large scale telemedicine: the Santa Catarina experience. Lat Am J Telehealth. 2009;1(3):364-77. Disponível em

20. World Health Organization. A universal truth: No health without a workforce. 
Geneva: WHO Press; 2013. 90 p. Disponível

em http://www.who.int/workforcealliance/ knowledge/resources/GHWA_AUniversalTruth Report.pdf.

\section{AGRADECIMENTOS}

Os autores agradecem a valiosa colaboração do Prof. Saulo Bortolon (UFES) na definição dos parâmetros a serem considerados para a escolha do conjunto de aquisição de imagens histopatológicas.

Também agradecem aos colegas que vêm testando e dando sugestões para melhoria da plataforma, em especial: Profa. Rejane Faria Ribeiro-Rotta (UFG), Prof. Viviane de Almeida Sarmento (UFBA), Profa. Manoela Domingues Martins (UFRGS) e Prof. Vinícius Carrard (UFRGS).

\section{ABSTRACT}

Collaborative multimedia platform to support oral diagnosis in teledentistry environments graphic and histopathologic images. The proposed solution is fully web-based, requiring only a web browser installed; it's not necessary to install any other software on the user's computer. As collaboration support, the actions performed by a user with mouse and keyboard are replicated and visible to the other connected user. This toolkit allows the discussion of disease via chat and/or videoconferencing. Telehealth's tools offer opportunities for assistance and education in Dentistry. By allowing realtime viewing and discussion of different aspects of a disease this technological solution has potential to facilitate: (1) exchange of information between health professionals at different levels of assistance and (2) distance tutoring at different levels of training (undergraduate, postgraduate and continuing education).

Descriptors: Teledentistry; Telemedicine; Diagnosis.

This paper presents a technological solution developed to enabling collaborative activities in Teledentistry environments. This is a platform composed by a set of tools for the management and sharing of clinical, radio- 